\title{
New records of Culicoides Latreille (Diptera: Ceratopogonidae) from Peruvian Amazonian region
}

\author{
Maria Luiza Felippe-Bauer ${ }^{1,6}$, Abraham Cáceres ${ }^{2}$, Cristiane Santos da Silva ${ }^{1}$, \\ William Valderrama-Bazan ${ }^{3}$, Antero Gonzales-Perez ${ }^{4}$ \& Janira Martins Costa ${ }^{5}$ \\ ${ }^{1}$ Laboratório de Diptera, Instituto Oswaldo Cruz-Fiocruz, \\ Av. Brasil, 4365, CEP 21040-900, Rio de Janeiro, RJ, Brazil \\ ${ }^{2}$ Divisão de Entomologia, Instituto Nacional de Saúde, Ministério da Saúde e \\ Instituto de Medicina Tropical "Daniel A. Carrión", \\ Universidade Nacional Maior de São Marcos, Lima, Peru \\ ${ }^{3}$ Serviço Nacional de Saúde Agrária, Ministério de Agricultura, Lima, Peru \\ ${ }^{4}$ Centro de Saúde Bagua Grande, Sub-região de Saúde Bagua, Amazonas, Peru \\ ${ }^{5}$ Departamento de Entomologia, Museu Nacional, \\ Universidade Federal do Rio de Janeiro - UFRJ, \\ Rio de Janeiro, RJ, Brazil \\ ${ }^{6}$ Corresponding author: Maria Luiza Felippe-Bauer, e-mail:mlfbauer@ioc.fiocruz.br
}

FELIPPE-BAUER, M.L., CÁCERES, G., SILVA, C.S., VALDERRAMA-BAZAN, W., GONZALES-PEREZ, A. \& COSTA, J.M. 2008. New records of Culicoides Latreille (Diptera: Ceratopogonidae) from Peruvian Amazonian Region. Biota Neotrop. 8(2): http://www.biotaneotropica.org.br/v8n2/en/abstract?article+bn00208022008.

Abstract: Ten species of Culicoides Latreille are reported for the first time from Peruvian Departments of Amazonas, Cajamarca, Loreto, Madre de Dios, Piura and San Martin. The synonymy and distribution of the species in the New World are given.

Keywords: Ceratopogonidae, neotropical bloodsucking midges, Culicoides, new records, Peru.

FELIPPE-BAUER, M.L., CÁCERES, G., SILVA, C.S., VALDERRAMA-BAZAN, W., GONZALES-PEREZ, A. \& COSTA, J.M. 2008. Novas ocorrências de Culicoides Latreille (Diptera: Ceratopogonidae) da Região Amazônica Peruana. Biota Neotrop. 8(2): http://www.biotaneotropica.org.br/v8n2/pt/abstract?article+bn0020 8022008 .

Resumo: Dez espécies de Culicoides Latreille são reportadas pela primeira vez para os departamentos peruanos de Amazonas, Cajamarca, Loreto, Madre de Dios, Piura e San Martin. A sinonímia e a distribuição das espécies para o novo mundo são fornecidas. 
Palavras-chave: Ceratopogonidae, maruins neotropicais, Culicoides, novos registros, Peru.

\section{Introduction}

The genus Culicoides Latreille presently contains 266 Neotropical species (Borkent \& Spinelli 2007). It is poorly known in Peru with 5 species originally described and only 16 more subsequently reported. In the Atlas of wing photographs of Neotropical species of Culicoides (Wirth et al. 1988) and in the catalog of the New World biting midges south of the United States of America (Borkent \& Spinelli 2000), C. efferus Fox and C. hitchocki Spinelli \& Wirth are cited as originally described from Peru. Wirth \& Blanton (1968) reported two species of the hylas species group, $C$. hylas Macfie and C. palpalis Macfie, from Peruvian Amazon region. Elliot et al. (1993) and Spinelli et al. (1993) recorded, respectively, C. venezuelensis Ortiz \& Mirsa and C. pseudodiabolicus Fox. Spinelli et al. (1996) reported 10 others species, as follows: C. belemensis Wirth \& Blanton, C. carpenteri Wirth \& Blanton, C. daviesi Wirth \& Blanton, C. eublepharus Macfie, C. fieldi Wirth \& Blanton, C. gabaldoni Ortiz, C. insignis Lutz, C. insinuatus Ortiz \& Leon, C. paucienfuscatus Barbosa and C. quasiparaensis Clastrier. Mercer et al. (2003) founded C. fluviatilis (Lutz) in Department of Loreto, Peru. C. paraensis (Goeldi) was previously mentioned by Barbosa (1947) and Wirth \& Felippe-Bauer (1989) from Iquitos and San Antonio, Department of Loreto. Its distribution in the New World and the description of more two species from Peru, belonging to Culicoides paraensis group, $C$. diversus and $C$. peruvianus, are made by Felippe-Bauer et al. (2003). Recently, Felippe-Bauer et al. (2005) described Culicoides (Diphaomyia) jurbergi from Departments of Amazonas, Cajamarca and Madre de Dios from Peruvian Amazon.

The purpose of this work is to provide the first records of 10 species of Culicoides from Peruvian Amazon Region.

\section{Material and Methods}

During an entomological survey in Peruvian Departments of Amazonas, Cajamarca, Loreto, Madre de Dios, Piura and San Martin, specimens of Culicoides are collected by CDC light trap or biting human. The specimens were mounted on microscope slides using the technique described by Wirth \& Marston (1968). For identification we used the taxonomic literatures cited for each species. The species listed below are included in subgeneric classification and species group according to Borkent \& Spinelli (2007). Specimens were deposited in Ceratopogonidae Collection's of Laboratory of Diptera, Instituto Oswaldo Cruz, Rio de Janeiro, as voucher material.

\section{Results and Discussion}

\section{New records from Peruvian Amazon region}

\section{Culicoides (Avaritia) pusillus Lutz, 1913}

Culicoides pusillus Lutz, 1913: 52 (male, female; Rio de Janeiro, Brazil; fig. wing); Macfie, 1938: 165 (redescript.; Trinidad and Tobago; fig. male genitalia); Floch \& Abonnenc, 1942b: 2 (Guyana records); Barbosa, 1947: 25 (Jamaica, Panama records); Macfie, 1948: 79 (Mexico records); Ortiz \& Mirsa, 1951: 603 (redescript.; Venezuela; figs. antenna, eyes, palpus, espermathecae, male genitalia, thorax); Ortiz \& Leon, 1955: 570 (Ecuador records); Wirth, 1955: 110 (Guatemala records); Forattini, 1957: 284 (redescript.; figs.); Forattini et al. 1958: 26 (larval habits; São Paulo, Brazil); Wirth \& Blanton, 1959: 292 (redescript.; figs.;
Panama distrib.); Williams, 1964: 463 (larval habitats; Trinidad and Tobago); Tikasingh, 1972: 447 (habits; Trinidad and Tobago); Wirth \& Blanton, 1973: 446 (Amazon records, Brazil); Aitken et al. 1975: 138 (Trinidad and Tobago records; wing photo); Winder \& Silva, 1972: 653 (larval habits; Brazil); Winder, 1977: 60 (larval habits; Brazil); Wirth et al. 1988: 15 (in Atlas; wing photo; distrib.); Borkent \& Wirth, 1997: 80 (in catalog); Ronderos \& Spinelli, 1998: 82 (Argentina records); Borkent \& Spinelli, 2000: 28 (in catalog); Mellor et al. 2000: 313 (epidemiology); Silva et al. 2001: 353 (records for Rio de Janeiro, Brazil); Soria et al. 2002: 320 (records for Pará, Brazil); Ronderos et al. 2003: 22 (record for the Yacyreta Dan lake area); Spinelli et al. 2005: 138 (in key, Argentina); Borkent \& Spinelli, 2007: 63 (in catalog).

Distribution: USA (Florida) to Central and South America to northeastern Argentina.

New records: PERU, Cajamarca, Provincia de San Ignacio, Distrito de La Coipa, Papayal Bajo, 13.II.2002, 1 female, biting human; Loreto, Provincia de Maynas, Distrito de Iquitos, 18/19. II.2001, 17/18.V.2001, 53 females, CDC light trap; Madre de Dios, Provincia de Tombopata, Distrito de Laberinto, Santa Rosa, 7/8.VIII.2002, 1 male, CDC light trap.

\section{Culicoides (Culicoides) hondurensis Spinelli \& Borkent, 2004}

Culicoides hondurensis Spinelli \& Borkent, 2004: 369 (female, male; El Salvador, Honduras; figs.); Borkent \& Spinelli, 2007: 64 (in catalog).

Distribution: El Salvador, Honduras, Peru.

New records: PERU, Cajamarca, Provincia San Ignacio: Distrito La Coipa, Papayal Bajo, 13.II.2002, 1 female, biting human; Distrito San Ignacio, Marizagua, IV.2000, 1 female, CDC light trap; Piura, Provincia Huancabamba, Distrito Huancabamba, VI.2000, 1 female, CDC light trap.

\section{Culicoides (Haematomyidium) debilipalpis Lutz, 1913}

Culicoides debilipalpis Lutz, 1913: 60 (female; Brazil; fig. wing); Macfie, 1937: 7 (female; redescript.; Trinidad and Tobago); Floch \& Abonnenc, 1942a: 3 (Guyana; figs. wing, palpus); Barbosa, 1952: 12 (Argentina, Brazil; fig. palpus); Forattini, 1957: 383 (in part.; redescript.; figs. eyes separation, antenna, palpus, wing, male genitalia; distrib.); Wirth \& Blanton, 1959: 442 (redescript.; figs.; Panama); Franca-Rodriguez, 1963: 67 (Uruguay); Messersmith, 1964: 339 (Viginia; breeding site); Smith \& Varnell, 1967: 520 (Florida; tree holes); Wirth \& Blanton, 1971a: 34 (redescript.; distrib.); Wirth \& Blanton, 1971b: 75 (khalafi as syn.; notes); Aitken et al. 1975: 119 (Trinidad and Tobago records); Blanton \& Wirth, 1979: 78 (redescript.; figs. eyes separation, antenna, palpus, wing, hind leg, spermathecae, male genitalia; distrib.); Vitale et al. 1981: 149 (notes; Panama); Borkent \& Wirth, 1997: 72 (in catalog, syn. lahillei); Spinelli \& Ronderos 1997: 304 (revalidation); Borkent \& Spinelli, 2000: 32 (in catalog); Ronderos et al. 2003: 22 (record for the Yacyreta Dan lake area); Spinelli et al. 2005: 141 (in key, Argentina); Trindade \& Gorayeb, 2005: 67 (records for Pará, Brazil); Borkent \& Spinelli, 2007: 66 (in catalog).

Culicoides ichesi Ronderos \& Spinelli, 1995: 77 (female; Argentina, Paraguay, Uruguay; figs. eyes separation, antenna, palpus, legs, spermathecae); Ronderos \& Spinelli, 1998: 81 (in key; record for the Yacyreta Dan lake area).

Culicoides khalafi Beck, 1957: 104 (male, female; Florida; fig. male genitalia).

Culicoides lahillei Spinelli \& Wirth, 1986: 62 (misident., in part., syn. debilipalpis); Wirth et al. 1988: 48 (in Atlas; wing photo; distrib.); Spinelli et al. 1989: 735 (in list; diurnal activity); Spinelli \& Ronderos, 1991: 91 (record for the Salto Grande Dan 
lake area); Spinelli \& Martinez, 1991: 171 (in key; Uruguay); Waller et al. 1990: 352 (redescript.; Guyana; wing photo).

Distribution: Widely distributed, from southeastern United States south to $35^{\circ} \mathrm{S}$ in the province of Buenos Aires, Argentina.

New records: PERU, Amazonas, Provincia de Utcubamba: Distrito de Cajaruro, El Aliso, 6.I.2002, 7 females, biting human; San Antonio, 22.IV.2001, 12.V.2001, 13.V.2001, 13.X.2001, 10.XI.2001, 11.XI.2001, 8.XII.2001, 7.I.2002, 24.II.2002, 15.III.2002, 17.III.2002, A.Gonzales-Perez, 77 females, biting human; Distrito de Jamalca, El Aserradero, 1.V.2001, 5.VI.2001, A.Gonzales-Perez, 6 females, biting human; Distrito Lonya Grande, El Rejo, 13.IV.2001, 4 females, biting human; Cajamarca, Provincia de San Ignacio: Distrito La Coipa, Papayal Bajo, 13.II.2002, 15 females, biting human; Distrito San Ignacio, Marizagua, IV.2000, 2 females, CDC light trap; Loreto, Provincia de Maynas, Distrito de Iquitos, 18/19.II.2001, 5 females, CDC light trap; Madre de Dios, Provincia Tombopata, Distrito Laberinto, Santa Rosa, 7/8.VIII.2002, 3 females, CDC light trap.

4. Culicoides (Haematomyidium) lahillei (Iches, 1906)

Ceratopogon lahillei Iches, 1906: 264 (female; Argentina, Chaco; figs., female, antenna, palpus, proboscis); Cavalieri \& Chiossone, 1966: 147 (sp. Inquir.); Spinelli \& Wirth, 1986: 62 (in key; syn. debilipalpis; wing photo erroneous); Wirth et al. 1988: 48 (in Atlas; syn. debilipalpis; wing photo erroneous, distrib.); Ronderos \& Spinelli, 1995: 80 (female redescript.; figs. eyes separation, antenna, palpus, wing); Borkent \& Wirth, 1997: 72 ( in catalog; debilipalpis as syn.); Spinelli \& Ronderos, 1997: 305 (male description); Ronderos \& Spinelli, 1998: 81 (in key; Argentina, Paraguay); Borkent \& Spinelli, 2000: 32 (in catalog); Soria et al. 2002: 320 (records for Pará, Brazil); Ronderos et al. 2003: 22 (record for the Yacyreta Dan lake area); Spinelli et al. 2005: 5 (in key; Argentina); Borkent \& Spinelli, 2007: 67 (in catalog).

Culicoides debilipalpis Costa Lima, 1937: 415 (in key; fig. palpus); Forattini, 1957: 383 (in part, at least specimens from Chaco, Argentina).

Distribution: Argentina (Chaco, Corrientes, Jujuy, Misiones), Peru, southeastern Brazil, Paraguay (Encarnacion, Capitan Meza).

New records: PERU, Amazonas, Provincia de Utcubamba: Distrito de Jamalca, El Aserradero, 21.X.2001, 6 females, biting human.

\section{Culicoides (Hoffmania) foxi Ortiz, 1950}

Culicoides foxi Ortiz, 1950a: 461 (male; Puerto Rico; figs.); Ortiz, 1951b: 4 (male, female; Venezuela; figs.); Fox, 1953: 888 (notes; Puerto Rico); Wirth \& Blanton, 1956: 309 (male, female; figs; distrib.); Forattini, 1957: 205 (redescrib.; figs; Brazil distrib.); Wirth \& Blanton, 1959: 283 (redescrib.; figs.; Panama distrib.); Fox \& Garcia-Moll, 1961: 120 (notes; Puerto Rico); Williams, 1964: 463 (larval habitats; Trinidad and Tobago); Aitken et al. 1968: 265 (habits; Trinidad and Tobago); Tikasingh, 1972: 447 (habits); Wirth, 1974: 24 (catalog); Wirth \& Blanton, 1973: 434 (Amazon records, Brazil); Aitken et al. 1975: 124 (Trinidad and Tobago records; wing photo); Ramirez Perez, 1984: 62 (Venezuela records); Greiner et al. 1984: 389 (Caribbean records); Spinelli \& Wirth, 1986: 51 (in key); Greiner \& Rawlins, 1987: 153 (Jamaica records); Lien \& Lu, 1987: 93 (redescript.; Bolivia; wing photo); Wirth et al. 1988: 16 (in Atlas; wing photo; distrib.); Greiner et al. 1989: 101 (Trinidad and Tobago records); Waller et al. 1990: 357 (notes; Guyana; wing photo); Spinelli \& Ronderos, 1991: 88 (notes; Argentina records); Spinelli et al. 1993: 41 (diagnosis; distrib.; figs.); Borkent
\& Wirth, 1997: 68 (in catalog); Borkent \& Spinelli, 2000: 34 (in catalog); Silva et al. 2001: 353 (records for Rio de Janeiro, Brazil); Soria et al. 2002: 320 (records for Pará, Brazil; Prov. Leoncio Prado, Peru); Ronderos et al. 2003: 22 (record for the Yacyreta Dan lake area); Laender et al. 2004: 145 (records for Minas Gerais, Brazil); Spinelli et al. 2005: 139 (in key, Argentina); Borkent \& Spinelli, 2007: 68 (in catalog).

Culicoides diabolicus Hoffman, 1925, of author, misident., in partim; Floch \& Abonnenc, 1942a: 2 (notes; Guyana; figs.).

Culicoides guttatus (Coquillett, 1904), of author, misident., in partim; Fox, 1948: 23 (female; Venezuela); Fox, 1949: 31 (male, female; Puerto Rico); Fox \& Kohler, 1950: 342 (biology; Puerto Rico).

Distribution: Mexico to Central and South America to Bolivia and Argentina; West Indies.

New records: PERU, Amazonas, Provincia de Utcubamba: Distrito de Cumba, Pitiana, 30/31.I.2001, A.G. Cáceres, 1 female, CDC light trap; Distrito de Lonya Grande, San Isidro, 14.IV. 2001, 1 male (CDC light trap), 2 females (biting human); Loreto, Provincia de Maynas, Distrito de Iquitos, 16.VI.2000, 2 females, biting human; Madre de Dios, Provincia de Tombopata, Distrito de Laberinto, Santa Rosa, 7/8.VIII.2002, 2,891 females, CDC light trap; San Martin, Provincia de San Martin, Distrito de Juan Guerra, El Porvenir, 24.II.2001, 2 females, CDC light trap.

\section{Culicoides (Hoffmania) ocumarensis Ortiz, 1950b}

Culicoides ocumarensis Ortiz, 1950b: 455 (male, female; Venezuela; figs); Ortiz \& Leon, 1955: 571 (notes; Ecuador; figs.); Wirth, 1974: 25 (in catalog); Lien \& Lu, 1987: 93 (notes; Bolivia; wing photo); Wirth et al. 1988: 18 (in Atlas; wing photo; distrib.); Spinelli et al. 1993: 63 (diagnosis; distrib.; figs.); Borkent \& Wirth, 1997: 77 (in catalog); Borkent \& Spinelli, 2000: 34 (in catalog); Borkent \& Spinelli, 2007: 69 (in catalog).

Culicoides diabolicus Hoffman, of authors, misident., in partim; Macfie, 1932: 488 (notes; Colombia record; habits); Wirth \& Blanton, 1956: 316 (syn. ocumarensis); Wirth \& Blanton, 1959: 280 (syn ocumarensis).

Culicoides lutzi Costa Lima, of authors, misident., in partim; Forattini, 1957: 238 (syn. ocumarensis).

Culicoides insignis Lutz, of authors, misident., in partim; Floch \& Abonnenc, 1942b: 2 (male; Guyana; figs.); Barbosa, 1944: 259 (male, female; Pernambuco, Brazil; figs.).

Culicoides recifei Barbosa, 1947: 25 (Pernambuco, Brazil; n. sp. for insignis Lutz of Barbosa, 1944); Barbosa, 1952: 20 (validation).

Culicoides filariferus Hoffman, of authors, misident., in partim; Aitken et al. 1975: 121 (syn. ocumarensis; Trinidad and Tobago records; wing photo).

Distribution: Widely distributed, from Florida to $35^{\circ} \mathrm{S}$ in Argentina.

New records: PERU, Madre de Dios, Provincia de Tombopata, Distrito de Laberinto, Santa Rosa, 7/8.VIII.2002, 17 females, CDC light trap; San Martin, Provincia de San Martin, Distrito de Juan Guerra, El Porvenir, 24.II.2001, 13 females, CDC light trap (12 females), biting sheep (1 female).

\section{Culicoides (Hoffmania) paraignacioi Spinelli, 1993}

Culicoides paraignacioi Spinelli, in Spinelli, Greiner \& Wirth, 1993: 66 (male, female; Belize, Costa Rica, Guyana, Colombia, Brazil; figs.); Borkent \& Wirth, 1997: 78 (in catalog); Borkent \& Spinelli, 2000: 34 (in catalog); Borkent \& Spinelli, 2007: 69 (in catalog). 
Distribution: Belize, Costa Rica, Guyana, Colombia, Brazil, Peru.

New records: PERU, Madre de Dios, Provincia de Tombopata, Distrito de Laberinto, Santa Rosa, 7/8.VIII.2002, 1 female, CDC light trap.

8. Culicoides (Hoffmania) plaumanni Spinelli, 1993

Culicoides plaumanni Spinelli, in Spinelli, Greiner \& Wirth, 1993: 69 (female; Argentina, Bolivia, Brazil; figs.); Borkent \& Wirth, 1997: 79 (in catalog); Borkent \& Spinelli, 2000: 34 (in catalog); Spinelli et al. 2005: 139 (in key; Argentina); Borkent \& Spinelli, 2007: 69 (in catalog).

Distribution: Brazil, Peru, Bolivia, Argentina.

New records: PERU, Loreto, Provincia de Maynas, Distrito de Iquitos, 14.VI.2001, 2 females, biting human; Madre de Dios, Provincia de Tombopata, Distrito de Laberinto, Santa Rosa, 7/8. VIII.2002, 139 females, CDC light trap.

9. Culicoides (Mataemyia) bricenoi Ortiz, 1951

Culicoides bricenoi Ortiz, 1951a: 445 (male; Venezuela; figs. male genitalia, wing); Ortiz, 1951b: 10 (in key); Fox, 1955: 223, 230 (in catalog); Ortiz \& Leon, 1955: 567, 570 (in key; female; Ecuador); Forattini, 1957: 348 (redescript.; figs. male genitalia, wing); Wirth \& Blanton, 1973: 428 (Amazon records, Brazil; figs. antenna, eye separation, legs, palpus, spermathecae, tibial comb, wing); Wirth et al. 1988: 36 (in Atlas; wing photo; distrib.); Borkent \& Wirth, 1997: 63 (in catalog); Borkent \& Spinelli, 2000: 35 (in catalog); Borkent \& Spinelli, 2007: 70 (in catalog).

Distribution: Venezuela, Ecuador, Brazil, Peru, Bolivia.

New records: PERU, Madre de Dios, Provincia de Tombopata, Distrito de Laberinto, Santa Rosa, 7/8.VIII.2002, 1 female, CDC light trap.

10. Culicoides leopoldoi Ortiz, 1951c, from fluviatilis species group

Culicoides leopoldoi Ortiz, 1951c: 579 (female; Venezuela; figs. antenna, palpus, thorax, wing); Ortiz \& Mirsa, 1951: 596 (male; Venezuela; figs. palpus, wing, genitalia); Ortiz \& Mirsa, 1952: 127 (redescript.; Venezuela, Panama records; figs. antenna, espermateca, palpus, wing); Forattini, 1957: 409 (redescript.; records for Pernambuco and Rio de Janeiro, Brazil; figs.); Wirth \& Blanton, 1959: 418 (redescript.; Panama distrib; figs.); Breeland, 1960: 165 (larval habitats; Panama); Williams, 1964: 463 (larval habitats; Trinidad and Tobago); Aitken et al. 1968: 265 (habits); Wirth \& Blanton, 1973: 441 (Amazon records, Brazil); Aitken et al. 1975: 131 (Trinidad and Tobago records; wing photo); Wirth et al. 1988: 45 (in Atlas; wing photo; distrib.); Borkent \& Wirth, 1997: 73 (in catalog); Ronderos \& Spinelli, 1998: 80 (in key; Argentina, Paraguay); Borkent \& Spinelli, 2000: 39 (in catalog); Silva et al. 2001: 353 (records for Rio de Janeiro, Brazil); Ronderos et al. 2003: 22 (record for the Yacyreta Dan lake area); Laender et al. 2004: 376 (records for Minas Gerais, Brazil); Spinelli et al. 2005: 140 (in key; Argentina); Borkent \& Spinelli, 2007: 72 (in catalog).

Distribution: Guatemala, Belize, Trinidad and Tobago to Bolivia and northeastern Argentina.

New records: PERU, San Martin, Provincia de San Martin, Distrito de Juan Guerra, El Porvenir, 24.II.2001, 1 male, CDC light trap.

\section{References}

AITKEN, T.H.G., WORTH, C.B. \& TIKASINGH, E.S. 1968. Arbovirus studies in Bush forest, Trinidad, W.I., September 1959-December 1964. III. Entomologic studies. Am. J. Trop. Med. Hyg. 17(2):253-268.
AITKEN, T.H.G., WIRTH, W.W., WILLIAMS, R.W., DAVIES, J.B. \& TIKASINGH, E.S. 1975. A review of the bloodsucking midges of Trinidad and Tobago, West Indies (Diptera: Ceratopogonidae). J. Entomol. (B) 44(2):101-144.

BARBOSA, F.A.S. 1944. "Culicoides insignis" Lutz, com a descrição do hipopígio (Diptera, Chironomidae). Rev. Bras. Biol. 4(2):259-261.

BARBOSA, F.A.S. 1947. Culicoides (Diptera: Heleidae) da região neotrópica. An. Soc. Biol. Pernambuco 7(1):3-30.

BARBOSA, F.A.S. 1952. Novos subsídios para o conhecimento dos Culicoides neotrópicos (Diptera: Heleidae). Thesis, Univ. Recife, Recife. 21p.

BECK, E. 1957. Two new species of Culicoides from Florida (Diptera: Heleidae). Florida Entomol. 40(3):103-105.

BLANTON, F.S. \& WIRTH, W.W. 1979. The sand flies (Culicoides) of Florida (Diptera: Ceratopogonidae). In Arthropods of Florida and Neighboring Land Areas Fla. Dept. of Agr. \& Consumer Ser. Div. Plant Ind., Gainesville 10:1-204.

BORKENT, A. \& SPINELLI, G.R. 2000. Catalog of the new world biting midges south of the United States of America (Diptera: Ceratopogonidae). Contrib. Entomol. Int. 4(1):1-107.

BORKENT, A. \& SPINELLI, G.R. 2007. Neotropical Ceratopogonidae (Diptera: Insecta). In Aquatic biodiversity in Latin America (J. Adis, J.R. Arias, G. Rueda-Delgado \& K.M. Wantzen, eds). Pensoft, SofiaMoscow. v. 4, 198p.

BORKENT, A. \& WIRTH, W.W. 1997. World species of biting midges (Diptera: Ceratopogonidae). Bull. Am. Mus. Nat. Hist. 233:1-257.

BREELAND, S.G. 1960. Observations on the breeding habitats of some Culicoides and other Heleidae in the Panama Canal Zone (Diptera). Mosquito News 20(2):161-167.

CAVALIERI, F. \& CHIOSSONE, I. 1966. Sobre el conocimiento actual de genero hematofago Culicoides Latreille, 1809, en la Argentina (Diptera: Ceratopogonidae). Physis 26(71):145-153.

COQUILLETT, D.W. 1904. A new Ceratopogon from Brazil. J. New York Entomol. Soc. 12:35-36.

COSTA-LIMA, A. 1937. Chave das especies de Culicoides da região neotrópica (Diptera: Ceratopogonidae). Mem. Inst. Oswaldo Cruz 32(3):411-422.

ELliot, A., CÁCERES, I., AGUILAR, N., PAREJA, E. \& ROMERO, G. 1993. Culicoides venezuelensis en el Peru. Rev. Per. Med. Trop UNMSM. 7:100-111

FELIPPE-BAUER, M.L., CÁCERES, A.G., SILVA, C.S., VALDERRAMABAZAN, W. \& GONZÁLES-PEREZ, A. 2003. Two new Culicoides of the paraensis group (Diptera: Ceratopogonidae) from the Amazon Region of Peru. Mem. Inst. Oswaldo Cruz 98(8):1051-1058.

FELIPPE-BAUER, M.L., CÁCERES, A.G., SILVA, C.S., VALDERRAMABAZAN, W. \& GONZÁLES-PEREZ, A. 2005. A new Culicoides (Diptera: Ceratopogonidae) of the subgenus Diphaomyia from Peru. Mem. Inst. Oswaldo Cruz 100(1):51-53.

FLOCH, H. \& ABONNENC, E. 1942a. Ceratopogonides hematophages de la Guyane Francaise. Publ. Inst. Pasteur de la Guyane et du Territ. de 1'Inini 37:1-10.

FLOCH, H. \& ABONNENC, E. 1942b. Ceratopogonides hematophages de la Guyane Francaise. II. Publ. Inst. Pasteur de la Guyane et du Territ. de l'Inini 49:1-5

FORATTINI, O.P. 1957. Culicoides da Região Neotropical (Diptera: Ceratopogonidae). Arq. Fac. Hig. Saude Publica Univ. São Paulo 11(2): $159-526$

FORATTINI, O.P., RABELLO, E.X. \& PATTOLI, D. 1958. Culicoides da região neotropical (Díptera: Ceratopogonidae). II. Observações sobre biologia em condições naturais. Arq. Fac. Hig. Saude Publica Univ. São Paulo 12(1):1-51.

FOX, I. 1948. Hoffmania, a new subgenus in Culicoides (Diptera: Ceratopogonidae). Proc. Biol. Soc. Wash. 61:21-28.

FOX, I. 1949. Notes on Puerto Rican biting midges or Culicoides (Diptera: Ceratopogonidae). Bull. Brooklyn Entomol. Soc. 44:29-34. 
FOX, I. 1953. Light trap studies on Culicoides in Puerto Rico. J. Econ. Entomol. 45:888-889.

FOX, I. 1955. A catalog of the bloodsucking midges of the Americas (Culicoides, Leptoconops, and Lasiohelea) with keys to the subgenera and nearctic species, a geographic index, and bibliography. J. Agric. Univ. Puerto Rico 39:214-285.

FOX, I. \& GARCIA-MOLL, I. 1961. The Culicoides of the international airport, Isla Verde, Puerto Rico, as shown by light traps. Mosquito News 21(2): 120-132.

FOX, I. \& KOHLER, C.E. 1950. Distribution and relative abundance of the species of biting midges or Culicoides in eastern Puerto Rico, as shown by light traps. Puerto Rico. J. Publ. Hlth. Trop. Med. 25:342- 349.

FRANCA-RODRIGUEZ, M.E. 1963. Primeros estudios sobre los Culicoides del Uruguay (Diptera: Ceratopogonidae). Rev. Urug. Patol. Clin. 1:65-70.

GREINER, E.C., ALEXANDER, F.C.M., ROACH, J., MOE, V., BORDE, G., TAYLOR, W.P., DICKISON, J. \& GIBBS, E.P.J. 1989. Bluetongue epidemiology in the Caribbean region: serological and entomological findings from a pilot sentinel system in Trinidad and Tobago. Med. Vet. Entomol. 3(2):101-105.

GREINER, E.C., GARRIS, G.I., ROLLO, R.T., KNAUSENBERGER, W.L., JONES, J.E. \& GIBBS, E.P.J. 1984. Preliminary studies on the Culicoides spp. as potencial vectors of bluetongue in the Caribbean region. Prev. Vet. Med. 2(1/4):389-399.

GREINER, E.C. \& RAWLINS, C. 1987. Culicoides spp. collected near ruminants in Jamaica and their relevance to bluetongue. J. Agric. Entomol. 4:153-156.

HOFFMAN, W.A. 1925. A review of the species of Culicoides of North and Central America and the West Indies. Am. J. Hyg. 5:274-301.

ICHES, L. 1906. Sobre cinco dipteros nuevos del Chaco austral. Bol. Minist. Agr. Rep. Argent. 6:262-273.

LAENDER, J.O., RIBEIRO, E.S., GOUVEIA, A.M.G., LOBATO, Z.I.P. \& FELIPPE-BAUER, M.L. 2004. Levantamento das espécies de Culicoides Latreille, 1809 (Diptera: Ceratopogonidae) encontradas nas mesorregiões Norte de Minas, Jequitinhonha e Vale do Mucuri, Minas Gerais, Brasil. Entomol. Vect. 11(1):145-157.

LIEN, JIH-CHING. \& LU, LIANG-CHEN. 1987. A small collection of biting midges of the genus Culicoides from Bolivia (Diptera: Ceratopogonidae). J. Taiwan Mus. 40:91-100.

LUTZ, A. 1913. Contribuição para o estudo das ceratopogoninas hematófagas do Brasil. Mem. Inst. Oswaldo Cruz 5 (1):45-72.

MACFIE, J.W.S. 1932. Some new or little-known Ceratopogonidae. Ann. Mag. Nat. Hist. Ser. 10(9):485-499.

MACFIE, J.W.S. 1937. Ceratopogonidae from Trinidad. Ann. Mag. Nat. Hist. 10(20):1-18.

MACFIE, J.W.S. 1938. Notes on Ceratopogonidae (Diptera). Proc. Royal Entomol. Soc. London. (B) 7: 157-166.

MACFIE, J.W.S. 1948. Some species of Culicoides (Diptera: Ceratopogonidae) from the States of Chiapas, Mexico. Ann. Trop. Med. Parasitol. 48:67-87.

MELLOR, P.S., BOORMAN, J. \& BAYLIS, M. 2000. Culicoides biting midges: Their role as arbovirus vectors. Ann. Rev. Entomol. 45:307-340.

MERCER, D.R., SPINELLI, G.R., WATTS, D.M. \& TESH, R.B. 2003. Biting rates and developmental substrates for biting midges (Diptera: Ceratopogonidae) in Iquitos, Peru. J. Med. Entomol. 40(6):807-812.

MESSERSMITH, D.H. 1964. The breeding site of Culicoides debilipalpis Lutz (Diptera: Ceratopogonidae) in Virginia. Mosquito News 24(3):339.

ORTIZ, I. 1950a. Informes sobre una nueva especie y lista de los machos cuyas genitalias son conocidas. Rev. Sanid. Asist. Soc. 15:461-465.

ORTIZ, I. 1950b. Estudios en Culicoides. IV. Revision de las especies americanas del subgénero Hoffmania (Fox 1948, con la descripcion de dos nuevas especies. Rev. Sanid. Asist. Soc. 15:437-460.
ORTIZ, I. 1951a. Estudios en Culicoides (Diptera:Ceratopogonidae). VI. Culicoides bricenoi n. sp. . Bol. Lab. Clin. Luis Razetti 16:442-448.

ORTIZ, I. 1951b. Estudios sobre Culicoides (Diptera:Ceratopogonidae). VII. Culicoides dominicii n. sp. y Clave para el reconocimiento de las especies venezolanas. Contr. Ocasionales Mus. Hist. Nat. La Salle 5:1-12.

ORTIZ, I. 1951c. Estudios en Culicoides (Diptera: Ceratopogonidae). Sobre los caracteres diferenciales entre Culicoides paraensis (Goeldi 1905), C. stellifer (Coquillett 1901), y C. lanei (Ortiz 1950). Descripcion de cuatro nuevas especies con la redescripcion de algunas otras poco conocidas. Rev. Sanid. Asist. Soc. 16:573-591.

ORTIZ, I. \& LEON, L.A. 1955. Los Culicoides (Diptera: Ceratopogonidae) de la republica del Ecuador. Bol. Inf. Cient. Nac. 67:564-590.

ORTIZ, I. \& MIRSA, M. 1951. Estudios en Culicoides. Descripcion de dos nuevas especies: Culicoides avilaensis y C. discrepans, y del macho de C. leopoldoi Ortiz, 1951. Redescripcion de C. limai Barretto, 1944, C. baueri Hoffman, 1925, C. lichyi Floch and Abonnenc, 1949, y C. pusillus Lutz, 1913. Rev. Sanid. Asist. Soc. 16:593-605.

ORTIZ, I. \& MIRSA, M. 1952. Sobre las especies americanas del genero "Culicoides" Latr. (Diptera: Ceratopogonidae) con una espermateca. Acta Cient. Venez. 3 (4):125-128.

RAMIREZ PEREZ, J. 1984. Revision de los dipteros hematofagos del genero Culicoides en Venezuela. Bol. Dir. Malariol. Sanid. Amb. 24:49-70.

RONDEROS, M.M. \& SPINELLI, G.R. 1995. Redescripcion de Culicoides lahillei y descripcion de Culicoides ichesi, n. sp. de la Argentina, Paraguay y Uruguay (Diptera: Ceratopogonidae). Neotropica 41(105-106):77-81.

RONDEROS, M.M. \& SPINELLI, G.R. 1998. Las espécies de Culicoides en el area de influencia de la represa de Yacyreta (Diptera: Ceretopogonidae). Rev. Soc. Entomol. Argent. 57(1-4):79-83.

RONDEROS, M.M., GRECO, N.M. \& SPINELLI, G.R. 2003. Diversity of biting midges of the genus Culicoides Latreille (Diptera: Ceratopogonidae) in the Area of the Yacyretá Dam Lake between Argentina and Paraguay. Mem. Inst. Oswaldo Cruz 98(1):19-24.

SILVA, C.S., FELIPPE-BAUER, M.L., ALMEIDA, E.H.G. \& FIGUEIREDO, L.R. 2001. Culicoides (Diptera: Ceratopogonidae) do estado do Rio de Janeiro, Brasil. I. Região Norte: Município de Campos do Goytacazes. Entomol. Vect. 8(3):349-358.

SMITH, W.W. \& VARNELL JR., J.H. 1967. Hydrogen ion concentration $(\mathrm{pH})$ as related to the occurrence and abundance of tree-holes dwelling Culicoides spp. (Diptera: Ceratopogonidae) in northern Florida. Mosquito News 27(4):519-521.

SORIA, S. J., FELIPPE-BAUER, M.L. \& OLIVEIRA, S.J. 2002. Lista das espécies de Ceratopogonidae (Diptera: Ceratopogonidae) do agroecossitema cacaueiro, depositadas na Coleção Entomológica do Instituto Oswaldo Cruz, Rio de Janeiro, Brasil. Entomol. Vect. 9(3):317-327.

SPINELLI, G.R. \& BORKENT, A. 2004. New species of Central American Culicoides Latreille (Diptera: Ceratopogonidae) with a synopsis of species from Costa Rica. Proc. Entomol. Soc. Wash. 106(2):361-395.

SPINELLI, G.R., FERNÁNDEZ, R., CARBAJAL, F., WATTS, D.M. \& WOOSTER, M.T. 1996. Nuevos registros de especies de Culicoides para el Peru (Diptera: Ceratopogonidae). Bol. Speit. 5(1):13-14.

SPINELLI, G.R., GREINER, E.C. \& WIRTH, W.W. 1993. The neotropical bloodsucking midges of the Culicoides guttatus group of the subgenus Hoffmania (Diptera: Ceratopogonidae). Contr. Am. Entomol. Inst. 27(3):1-91.

SPINELLI, G.R. \& MARTINEZ, M.E. 1991. The genus Culicoides in Uruguay (Diptera: Ceratopogonidae). Insecta Mundi 5(3-4):175-179.

SPINELLI, G.R. \& RONDEROS, M.M. 1991. Los polvorines del genero Culicoides en area de influencia de la represa de Salto Grande (Diptera: Ceratopogonidae). Neotropica 37(98):83-94.

SPINELLI, G.R. \& RONDEROS, M.M. 1997. Description of the male of Culicoides lahillei and revalidation of Culicoides debilipalpis (Diptera: Ceratopogonidae). Trans. Am. Entomol. Soc. 123(4):303-309.

SPINELLI, G.R., RONDEROS, M.M. \& BALSEIRO, E.G. 1989. Analisis de la actividad diaria de dipterous nematoceros en Punta Lara (Pdo. 
de Enseada, Prov. de Buenos Aires). II. Ceratopogonidae, con la descripcion de una especie nueva del género Atrichopogon. Limnobios 2(10):733-737.

SPINELLI, G.R., RONDEROS, M.M., DÍAZ, F. \& MARINO, P.I. 2005. The bloodsucking biting midges of Argentina (Diptera: Ceratopogonidae). Mem. Inst. Oswaldo Cruz 100(2):137-150.

SPINELLI, G.R. \& WIRTH, W.W. 1986. Clave para la identificacion de las especies del género Culicoides Latreille presentes al sur de la cuenca amazonica. Nuevas citas y notas sinonimicas (Diptera: Ceratopogonidae). Rev. Soc. Entomol. Argent. 44(1):49-73.

TIKASINGH, E.S. 1972. Seasonal and diurnal activities of four species of Trinidadian Culicoides (Diptera: Ceratopogonidae). Mosquito News 32(3): 447-452.

TRINDADE, R.L. \& GORAYEB, I.S. 2005. Maruins (Ceratopogonidae: Diptera) do estuário do rio Pará e do litoral do estado do Pará, Brasil. Entomol. Vect. 12(1):61-74.

VITALE, G.C., WIRTH, W.W. \& AITKEN, T.H.G. 1981. New species and records of Culicoides reared from arboreal habitats in Panama, with a synopsis of the debilipalpis group (Diptera: Ceratopotonidae). Proc. Entomol. Soc. Wash. 83(1):140-159.

WALLER, J., LANCELOT, R., LEFEVRE, P.C. \& KREMER, M. 1990. Note faunistique sur les Culicoides de Guyane (Diptera: Ceratopogonidae). Rev. Elev. Med. Vet. Pays Trop. 43(3):349-364.

WILLIAMS, R.W. 1964. Observations on habitats of Culicoides larvae in Trinidad, W.I. (Diptera: Ceratopogonidae). Ann. Entomol. Soc. Am. 57:462-466.

WINDER, J.A. 1977. Field observations on Ceratopogonidae and other Diptera: Nematocera associated with cocoa flowers in Brazil. Bull. Entomol. Res. 67:57-63.

WINDER, J.A. \& SILVA, P. 1972. Cacao pollination: Microdiptera of cacao plantations and some of their breeding places. Bull. Entomol. Res. 61:651-655.
WIRTH, W.W. 1955. Report on a collection of biting midges of the genus Culicoides from Guatemala (Diptera: Heleidae). Proc. Entomol. Soc. Wash. 57(3):109-117.

WIRTH, W.W. 1974. A catalogue of Diptera of the Americas south of the United States. Ceratopogonidae. Mus. Zool. Univ. São Paulo 14:1-89.

WIRTH, W.W. \& BLANTON, F.S. 1956. Studies in Panama Culicoides VIII. The Neotropical species of the guttatus group of the subgenus Hoffmania (Ditera: Heleidae). Proc. Entomol. Soc. Wash. 58(6):305-326.

WIRTH, W.W. \& BLANTON, F.S. 1959. Biting midges of the genus Culicoides from Panama (Diptera: Heleidae). Proc. U.S. Nat. Mus. 109(3415):237-482.

WIRTH, W.W. \& BLANTON, F.S. 1968. A Revision of the Neotropical biting midges of the hylas group of Culicoides (Diptera: Ceratopogonidae). Fla. Entomol. 51(4):201-215.

WIRTH, W.W. \& BLANTON, F.S. 1971a. New neotropical sandflies of the Culicoides debilipalpis group (Diptera: Ceratopogonidae). Proc. Entomol. Soc. Wash. 73(1):34-43.

WIRTH, W.W. \& BLANTON, F.S. 1971b. New species and synonymy of Florida Culicoides (Diptera: Ceratopogonidae). Fla. Entomol. 54(1):73-78.

WIRTH, W.W. \& BLANTON, F.S. 1973. A review of the maruins or biting midges of the genus Culicoides (Diptera: Ceratopogonidae) in the Amazon Basin. Amazoniana 4(4):405-470.

WIRTH, W.W. \& FELIPPE-BAUER. M.L. 1989. The neotropical biting midges related to Culicoides paraensis (Diptera: Ceratopogonidae). Mem. Inst. Oswaldo Cruz 84(Supl. 4):551-565.

WIRTH, W.W. \& MARSTON, N. 1968. A method for mounting small insects on microscope slides in Canada Balsam. Ann. Entomol. Soc. Am. 61(3):783-784.

WIRTH, W.W., DYCE, A.L. \& SPINELLI, G.R. 1988. An Atlas of wing photographs, with a summary of the numerical characters of the neotropical species of Culicoides (Diptera: Ceratopogonidae). Contr. Am. Entomol. Inst. 25(1):1-72. 